\title{
Sharp scattering spectra induced brilliant and directional structural colors
}

\author{
Zhipeng Meng, Yue Wu, Shufen Zhang and Suli Wu
}

\begin{abstract}
Structural color materials, which generate colors through the interaction between light and nano-microstructures, have always been research hotspots in the fields of display, anticounterfeiting and stimuli-responsive materials. Structural colors based on scattering have received increasing attention due to their wider viewing angles than that originating from the specular reflection of photonic crystals. However, the wide scattering spectrum of an amorphous structure leads to lower purity and brightness of the appeared colors. Few researchers have focused on the scattering of ordered structures due to their strong reflection and diffraction in the visible regions. In this work, by building ordered films (OFs) using $\mathrm{SiO}_{2}$ spheres (refractive index $n=1.46$ ) with a diameter of $300-500 \mathrm{~nm}$, for the first time, sharp scattering spectra with narrow full width at half-maximum (FWHM, $24 \mathrm{~nm}$ ) were generated. Importantly, under ambient light, brilliant colors covering the entire visible region can be observed, and a formula was proposed to calculate the scattering spectra of OFs. Moreover, rainbow structural color was realized under irradiation of the nonparallel light, and fullspectrum structural color patterns were fabricated using building blocks with a single particle size by a spraying method. Finally, a composite structure was constructed to explore possibilities in the field of flexible transparent displays.
\end{abstract}

Keywords: sharp scattering, structural colors, $\mathrm{SiO}_{2}$ spheres, transparent display, ordered or disordered assembly

\section{INTRODUCTION}

Accurate control of color generation and application has always been the focus of display research. Structural colors originating from light interference [1], diffraction [2], or the scattering [3,4] effect in microphysical structures instead of pigments or dyes have recently attracted widespread attention. These colors have a unique ability to manipulate light with specific wavelengths [5] and have excellent photobleaching resistance [6-8]. In nature, most bright colors are produced by special microstructure to control the flow of light $[9,10]$ (vivid colors of butterflies [11], bird feathers or rainbows [12]). The structural colors of photonic crystals (PCs) have been intensively studied in many practical applications because of their ability to reflect light with certain wavelengths [13-15]. However, the widening and weakening of the photonic bandgap (PBG) caused by structural defects hinder the large-scale applications of PCs. Different from the traditional soft monomer strategy, Wu et al. [16] presented a proof-ofconcept to achieve robust PCs with large-scale crack-free arrays and good flexibility using hydrophobic soft monomers. Recently, as an alternative way to generate structural colors, light scattered by nanoparticles has received more attention [17,18]. Different from PCs, structural colors originating from scattering have wide viewing angles, and their observation is not necessary at specular angles of incident light. For example, color generation through the scattering effect has been demonstrated from many polydisperse, near micron-sized $\mathrm{TiO}_{2}$ particles [19]. Bai et al. [20] developed rapid, large scale fabrication of full-spectrum structural color patterns with wide viewing angles by amorphous colloidal arrays. Noniridescent structural colors mimicking the optical properties of bird feathers were also achieved successfully by Forster et al. [21]. However, these scattering structural colors have low color purity and brightness due to the week reflectance and wide FWHM determined by their amorphous structures. Furthermore, the realization of full-spectrum colors needs particles with different sizes to scatter different wavelengths of light, which limits their application in the color display field to some extent. Due to strong reflection and diffraction in the visible region, few researchers have focused on the scattering spectra of

State Key Laboratory of Fine Chemicals, Dalian University of Technology, Dalian 116024, China

* Corresponding author (email: wusuli@dlut.edu.cn) 
ordered structures. For example, PCs assembled by $\mathrm{SiO}_{2}$ spheres of $200-300 \mathrm{~nm}$ show highly angle-dependent reflected colors under light irradiation.

The Mie scattering theory proposed by Gustav Mie provides a basic scattering principle for spherical particles [22]. Many studies on the scattering effect of nanoparticles with visible and near-infrared light have been reported [23-26]. According to the Mie theory, when the light wavelength inside the particle becomes comparable to the particle's diameter $(D)$, the resonant magnetic dipole response resulting from the coupling of incoming light to the circular displacement currents of the electric field will occur [27]. This can be roughly described by the formula of $D \approx \lambda / n$, where $n$ is the refractive index of the particle, and $\lambda$ is the wavelength of light. This means that if the particle size is large enough, Mie scattering can be effectively realized by low-refractive-index materials. For example, hollow $\mathrm{SiO}_{2}$ spheres with thin shell thickness were reported to show blue, green and pale violet scattering colors in a dark background [24], but it is still challenging to obtain brilliant colors covering the entire visible range under ambient light.

Here, monodisperse $\mathrm{SiO}_{2}(n=1.46)$ colloidal microspheres with particle size over $300 \mathrm{~nm}$ were selected to generate structural colors based on the scattering effect. Ordered structures of $\mathrm{SiO}_{2}$ spheres were prepared by a dip-coating method to increase their Mie scattering effect. Under ambient light, bright colors can be observed easily in nonspecular angles due to their sharp scattering spectra with narrow FWHM and relatively high scattering intensity. Meanwhile, rainbow scattering was also achieved under irradiation of a light emitting diode (LED) source. Furthermore, the amorphous structures of $\mathrm{SiO}_{2}$ spheres prepared by a spraying method were also investigated, similar rainbow colors appeared with the same irradiation, and a full-spectrum structural color pattern was realized using building blocks with a single particle size based on the Mie scattering. Finally, the sandwich structure fabricated by polyethylene terephthalate (PET) substrate, a patterned amorphous $\mathrm{SiO}_{2}$ middle layer and polydimethylsiloxane (PDMS) packaging materials was applied to make the flexible, transparent display possible.

\section{EXPERIMENTAL SECTION}

\section{Preparation of monodisperse $\mathrm{SiO}_{2}$ colloidal spheres}

Monodisperse $\mathrm{SiO}_{2}$ colloidal spheres were prepared by a modified Stober method. The size of $\mathrm{SiO}_{2}$ colloidal spheres can be controlled by tuning the amount of aqu- eous ammonia. Silica spheres with diameters of 306, 340, $360,410,435$ and $460 \mathrm{~nm}$ were obtained. Products were collected by centrifugation and washed several times with ethanol. The obtained solid product was vacuum dried. Then, a $\mathrm{SiO}_{2}$ suspension (8 $\mathrm{wt} \%$ or $6 \mathrm{wt} \%$ ) in absolute ethanol was prepared by dispersing spheres through sonicating for $24 \mathrm{~h}$ before dip-coating.

\section{Preparation of monodisperse PMMA colloidal spheres}

Monodisperse polymethyl methacrylate (PMMA) microspheres were synthesized by emulsion polymerization. First, $100 \mathrm{~mL}$ of deionized water was added to a $250-\mathrm{mL}$ three-necked flask, and the oil bath was set at a temperature of $40^{\circ} \mathrm{C}$. The mechanical stirring device was introduced into the middle port, nitrogen was supplied to the side port, and circulating cooling water was added to the other side to prevent water evaporation during the reaction. Then, $10-30 \mathrm{~g}$ of MMA was added to the reaction system and heated to $85^{\circ} \mathrm{C}$ for at least $30 \mathrm{~min}$ under $300 \mathrm{rpm}$ stirring to form an emulsion. Then, $0.1-$ $0.3 \mathrm{~g}$ of the potassium peroxydisulfate was added into the reaction system and reacted for $5 \mathrm{~h}$ under nitrogen atmosphere. The product could be used directly without further processing.

\section{Fabrication of ordered films (OFs) with different thicknesses}

The substrates for building the photonic structures are glass slides which are washed with water before use. The dip-coating process was carried out at a temperature of $35^{\circ} \mathrm{C}$ using a dip-coater from HeatMent (SYDC- $100 \mathrm{H}$ DPI, SAN-YAN Instrument Co. Ltd., Shanghai). The pulling rate was set at $2 \mu \mathrm{m} \mathrm{s}^{-1}$. Cycle times were set from 1 to 3 to obtain different sample thicknesses with $8 \mathrm{wt} \%$ and $6 \mathrm{wt} \% \mathrm{SiO}_{2}$ suspensions.

\section{Fabrication of patterned amorphous films (AFs)}

Spray coating of the amorphous $\mathrm{SiO}_{2}$ or PMMA layer was performed using an airbrush possessing a nozzle with a size of $0.2 \mathrm{~mm}$, which was driven by a 0.7 -bar gas pump, and the distance between the airbrush and the substrate was kept at $5 \mathrm{~cm}$. The thickness of the amorphous structure was controlled by tuning the number of spraying cycles. The patterns were realized using a mask during the spray-coating process. The part without the mask was covered by disordered $\mathrm{SiO}_{2}$ or PMMA microspheres.

\section{Characterizations}

The reflection, scattering and transmission spectra of the 
ordered structures of $\mathrm{SiO}_{2}$ were recorded using a Hitachi U-4100 spectrophotometer. The microscopic sample morphology was characterized using a Nova Nano SEM 450 field emission scanning electron microscope. All the digital photos of the structural colors of the ordered and amorphous films were taken with a digital camera (iPhone).

\section{RESULTS AND DISCUSSION}

As the most common colloidal microspheres used to artificially construct microstructures, monodisperse $\mathrm{SiO}_{2}$ microspheres were adopted here as building blocks to scattering light. Based on the equation $D \approx \lambda / n$, monodisperse $\mathrm{SiO}_{2}$ spheres with different particle sizes (360, 410 and $460 \mathrm{~nm}$ ) were first synthesized to obtain different structural colors. The synthesized spheres were selfassembled into OFs on two sides of the glass slide by the dip-coating method (Fig. 1a). We defined the OFs constructed by 360,410 and $460 \mathrm{~nm} \mathrm{SiO}_{2}$ spheres as OF (360), OF (410) and OF (460), respectively. Excitingly, under the illumination of ambient collimated light (Fig. 1c), OFs with different particle sizes (I: $360 \mathrm{~nm}$, II: $410 \mathrm{~nm}$, III: $460 \mathrm{~nm}$ ) exhibit brilliant structural colors in the white background, which is better than most of the reported structural materials, especially for the scattered sample. Taking OF (410) as an example, the corresponding plane and cross-sectional scanning electron microscopy ( SEM ) images are shown in Fig. 1b, and the inset of the two-dimensional Fourier transform (2D-FFT) pattern with hexagonal peaks clearly indicates the longrange ordered structure of the OF (410).

To gain further insight into the scattering colors of the ordered array of $\mathrm{SiO}_{2}$ spheres, photographs of the scattered colors of OF (360), OF (410) and OF (460) were taken at different viewing angles under ambient light. It is obvious that the structural color has strong viewing-angle-dependence due to the anisotropy of its excellent crystal structure (Fig. 1d). Each ordered film shows different brilliant colors with the change in the viewing angles. Specifically, ordered structures consisting of 410 and $460 \mathrm{~nm} \mathrm{SiO}_{2}$ spheres exhibit vivid structural colors covering the whole visible region when varying the viewing angles. Video $S 1$ clearly shows the color changing process of OF (410) in a rotating sample under ambient light. It is worth mentioning that the angle-dependent properties of the scattered structural color are different from that of PCs. The angle-dependent color of PCs refers to the color variation observed under specular angle a
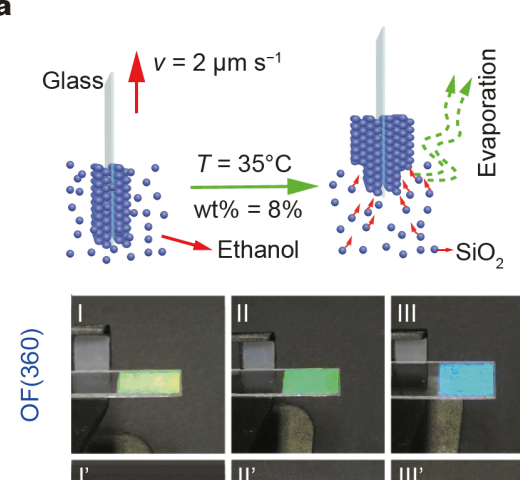

鎄
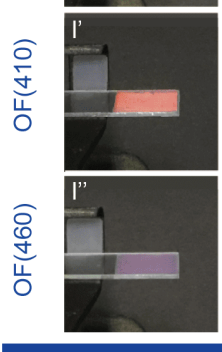

$\theta=15^{\circ}$

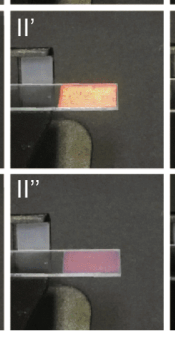

$25^{\circ}$ b
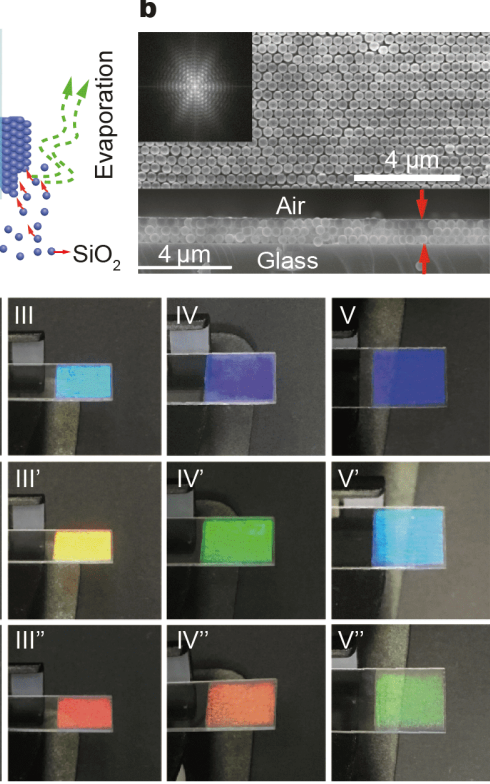

$35^{\circ}$
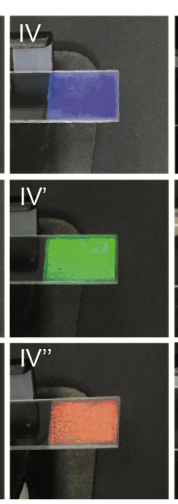

$45^{\circ}$
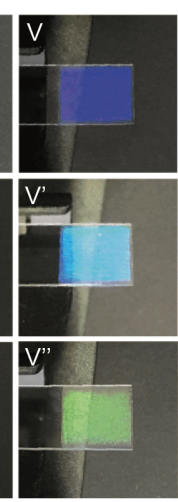
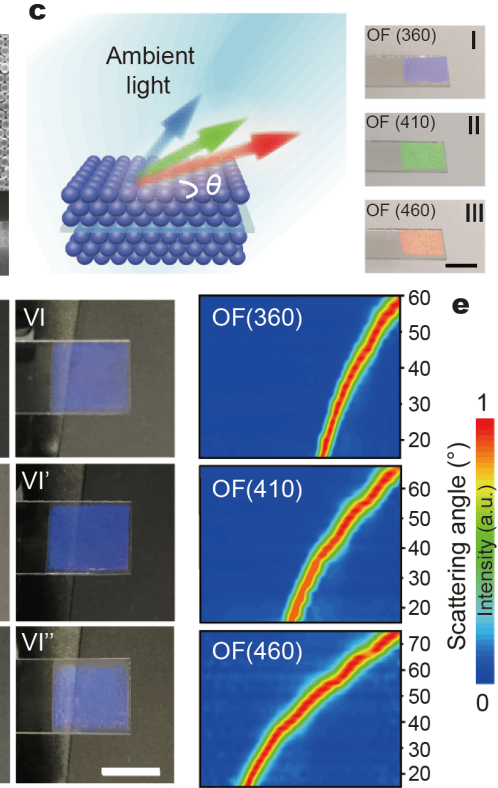

$65^{\circ}$

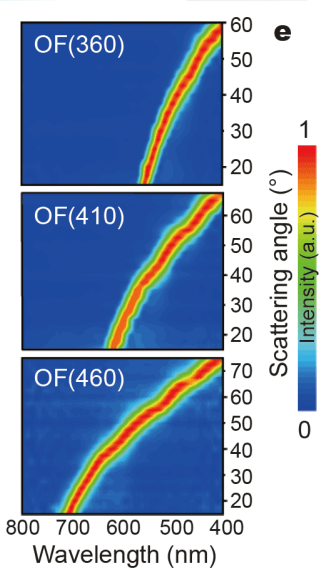

Figure 1 (a) Schematic illustration of the fabrication process of OFs by the consecutive dip-coating method. (b) Plane and single side cross-sectional SEM image of the OFs fabricated by $410 \mathrm{~nm}$ of $\mathrm{SiO}_{2}$. Inset is the 2D-FFT pattern. (c) Schematic illustrations of the angle-resolved iridescent colors backward scattered by the OFs under ambient collimated illumination. (d) Backward scattering images of the OFs assembled with $\mathrm{SiO}_{2}$ microspheres of 360,410 and $460 \mathrm{~nm}$ at different view angles from $15^{\circ}-65^{\circ}$. (e) Color-filled contour maps of the scattering spectra of the corresponding OFs (each scattering spectrum was collected every $2.5^{\circ}$ with an incident light angle of $45^{\circ}$ ). 
of incident light, which means that varied color is obtained by simultaneously changing the incident and detection angles. However, the scattered color variation in Fig. $1 \mathrm{~d}$ is viewed only by changing the viewing angle with the fixed incident light. Furthermore, at the same angles, the colors are redshifted with the increasing diameter of the building blocks, which highlights the importance of particle size with respect to light in generating different structural colors.

According to previous reports [28-30], directional scattering is an important feature of Mie scattering. Here, the forward and backward scattering spectra of the three OFs were measured with the detecting angle at $35^{\circ}$ (Fig. S1a-c). All the spectra agree well with the observed structural color, indicating that the colors originate from the scattering of the ordered structure. Different from the traditional multipeak in the Mie scattering spectrum resulting from higher order multipoles $[26,27]$ in the $3 \mathrm{D}$ ordered structure of $\mathrm{SiO}_{2}$, the assembled particles lead to enhanced coupling of the magnetic dipole. The dipole appears as a strong single peak in the scattering spectrum, and the scattering peaks of other higher-order modes are suppressed. At the same time, the reflection spectra of all the OFs are located in the near infrared (NIR) region, proving that structural colors are not related with the ordered structure reflection (Fig. S1d-f). Moreover, compared with the forward and backward spectra of each film, one can see that when the angle between the detector and the sample is fixed, the forward and backward scattering spectra of each film have very similar peak positions and shapes, which means good symmetric structural colors of the sample. In addition, the scattering spectra redshift from 510 and 570 to $650 \mathrm{~nm}$ when the diameter of $\mathrm{SiO}_{2}$ spheres increases from 360 and 410 to $460 \mathrm{~nm}$, which agrees well with the digital photos of cyanblue, yellow and red in the third column of Fig. 1d. Due to the difficulty in detecting backward scattering spectra near incident angles in the test system, forward scattering spectra were applied here to describe the angle-dependent colors of the OFs in the later part.

Consistent with the structural colors at different viewing angles, the scattering spectra of each sample redshift with an increasing detecting angle (Fig. 1e). It is noteworthy that for the OFs built with 410 and $460 \mathrm{~nm} \mathrm{SiO}_{2}$ spheres, their scattering spectra can cover the whole visible region by changing the detecting angle, which strongly demonstrates the decisive role of particle size in Mie scattering. To further confirm the generality of sizeand detection angle-dependent properties of the scattering spectra, 306, 330 and $435 \mathrm{~nm} \mathrm{SiO}{ }_{2}$ spheres, as shown in SEM images in Fig. S2a, were also synthesized and selfassembled into the ordered structure by the same process. The corresponding scattering spectra listed in Fig. S2b demonstrate the same size and detection angle dependence. Digital images were also captured at $35^{\circ}$, as shown in Fig. S2c, and the vivid purple, cyan and red colors show good scattering ability of OF (306), OF (330) and OF (435).

For scattering structural colors, there is no doubt that the sample thickness has a significant effect on the colors at different scattering directions, whether for ordered or amorphous structures [18,31,32]. Here, taking the film assembled by $360 \mathrm{~nm} \mathrm{SiO}{ }_{2}$ spheres as an example, the unilateral cross-sectional SEM images in Fig. 2a-d indicate that highly ordered films with different thicknesses were successfully achieved. The forward and backward scattering spectra of these samples were measured at a detecting angle of $35^{\circ}$ under an incident angle of $45^{\circ}$ (Fig. 2e, f). It is clear that when the film is only composed of one layer of $\mathrm{SiO}_{2}$ spheres on one side of the glass, both the forward and backward scatterings are very weak (black line in Fig. 2e, f), because the incomplete coverage of $\mathrm{SiO}_{2}$ spheres on the glass substrate in this situation reduces the scattering intensity. For the other three samples, the forward scattering intensity (Fig. 2e) gradually decreases as the thickness of OF (360) increases. In contrast, the backward scattering intensity slightly increases at the same detecting angle (Fig. 2f), which can be described by the effective light penetration layers of the ordered structure. According to Yuan's report [33], the scattering light can only pass through 12 layers of the assembled structure. In the backward scattering process, light needs to pass through the doubled thickness of the sample (Fig. 2g). Here, OF (360)-b prepared by the dipcoating method has 6 layers of ordered spheres on two sides of the glass slide, and the backward scattering light passes through 12 layers of spheres. For samples $\mathrm{c}$ and d, although they have thicker assembly layers (12 and 16 layers, respectively), the effective number of layers for scattering is the same (theoretically 12 layers) as that of sample b. Hence, OF (360)-b, c and d have the similar scattering intensity in the backward scattering mode. The digital images in Fig. S3c show the backward scattering structural colors of the films with different thicknesses, demonstrating their good manipulating ability for light, except for the OF (360)-a, which shows dim colors due to weak scattering intensity. Meanwhile, the forward and backward scattering spectra of the samples were also compared in Figs S1a and S3a, b. Obviously, for each sample, the scattering spectra at different directions are 

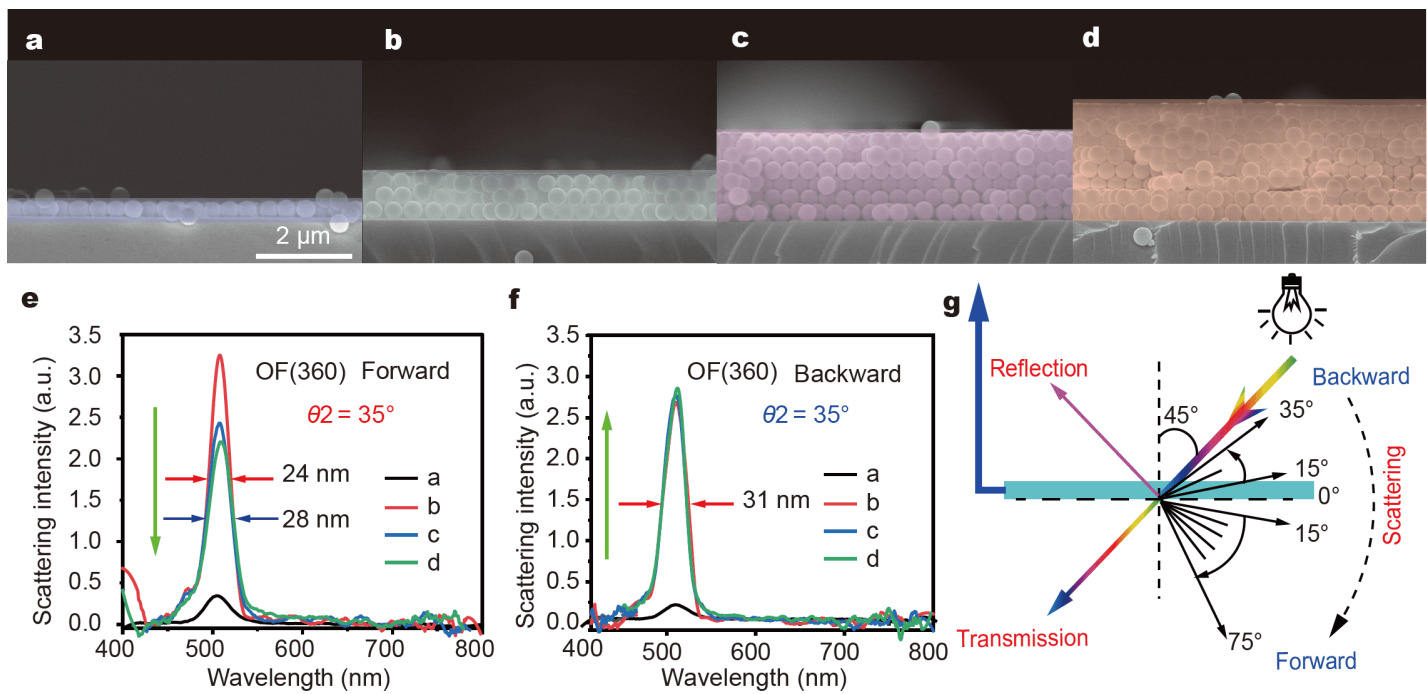

Figure 2 (a-d) Typical single side cross-sectional SEM images of the OFs fabricated by $360 \mathrm{~nm}$ of $\mathrm{SiO}_{2}$ spheres with different thicknesses. Samples with different assembly layers were named OF (360)-a, OF (360)-b, OF (360)-c and OF (360)-d. Scale bar: $2 \mu \mathrm{m}$. (e, f) Forward and backward scattering structural spectra of the structures of $(\mathrm{a}-\mathrm{d})$ with incident light angle $\theta 1=45^{\circ}$, and detection angle $\theta 2=35^{\circ}$. FWHM of forward scattering spectra: $\mathrm{OF}(360)-\mathrm{b}=24 \mathrm{~nm}$, OF $(360)-\mathrm{c}=28 \mathrm{~nm}$. Backward scattering spectra: OF $(360)-\mathrm{b}=31 \mathrm{~nm}$. $(\mathrm{g})$ Optical path diagram of the interaction between light and the prepared samples.

similar in shape and position. For thinner films, the forward scattering is slightly greater than the backward scattering (Fig. S1a). While for the thicker film (6 and 8 layers of spheres on one side of glass), the backward scattering is slightly greater than the forward scattering. To obtain higher forward scattering, the film with 3 layers of spheres on the side of glass was adopted in later experiments.

To explain the effect of $\mathrm{SiO}_{2}$ film thickness on the scattering effect, the absorption and transmission spectra of these film were also recorded, as shown in Fig. $\mathrm{S}$. $\mathrm{SiO}_{2}$ itself has nearly no absorption in the visible region (black line in Fig. S4a). When an electromagnetic wave interacts with the $\mathrm{SiO}_{2}$ film, the wave will be selectively reflected, scattered and transmitted, as illustrated in Fig. 2g. The transmittance of the films decreases with the increase in the thickness, which contributes to reduction of the forward scattering intensity of the thicker film. On the other hand, a wide valley in the visible region (blue dotted frame section in Fig. S4b) appeares in the transmission spectra, which is in accordance with the result displayed in the forward scattering spectra of OF (360), as shown in Fig. 1e. Importantly, different from the general scattering spectrum, each sample displays sharp scattering spectra with narrower FWHM (Fig. 2e, f) due to the resonance coupling effect of the ordered structure. The sharp scattering spectra give the samples brilliant structural colors with high purity, which guarantees their great potential applications in display devices.

In a further set of experiments, we investigated the effect of different incident angles on the forward scattering at certain detecting angles. For convenience, $\theta 2$ was fixed at $35^{\circ}$, and the specific optical path acquisition system is shown in Fig. 3b. OF (360) and OF (435) were taken as examples to investigate the incident angledependent property of the structural colors based on Mie scattering. The scattering spectra listed in Fig. 3a, c show that the realization of OF structural colors can be finely and easily tuned by changing the direction of $\theta 1$. When the incident angle varies from $10^{\circ}$ to $65^{\circ}$, the scattering spectra of OF (360) blueshift from 600 to $410 \mathrm{~nm}$. For OF (435), when the incident angle varies from $10^{\circ}$ to $75^{\circ}$, its scattering spectra changes from 720 to $430 \mathrm{~nm}$, covering the whole range of visible light. Obviously, the range of the scattering spectra of OF (435) is larger than that of OF (360) by approximately $100 \mathrm{~nm}$, which indicates the strong size-dependent ability of $\mathrm{SiO}_{2}$ films to manipulate visible light. These consecutively changed structural colors observed at one direction are essentially different from the previously reported structural color of PCs, in which the structural color must be observed at a specular angle, and the color change occurs by simultaneously changing the incident and detecting angles. Importantly, the fine-tuning property of the scattering structural color at a fixed viewing direction by simply changing the incident angle may provide options to generate full-spec- 


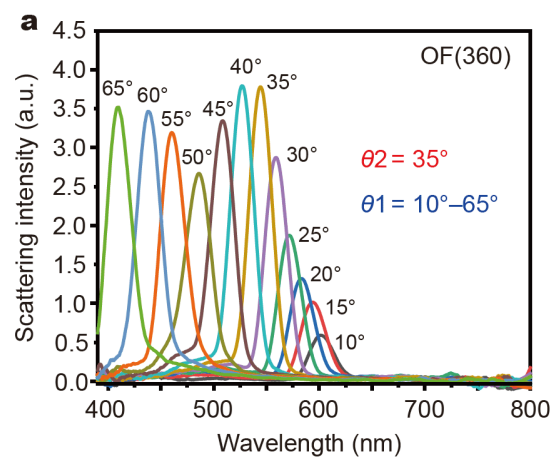

d

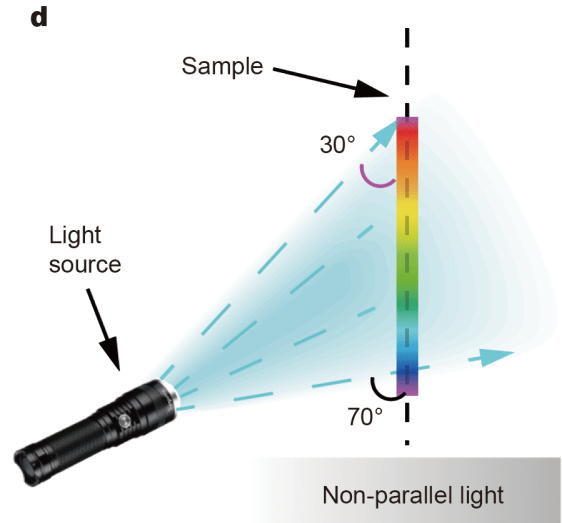

b

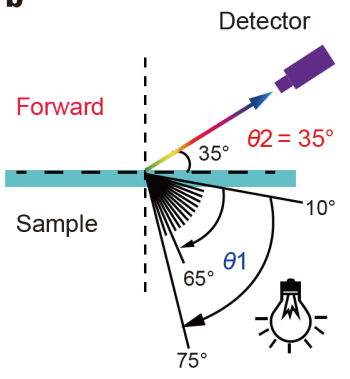

Light Source

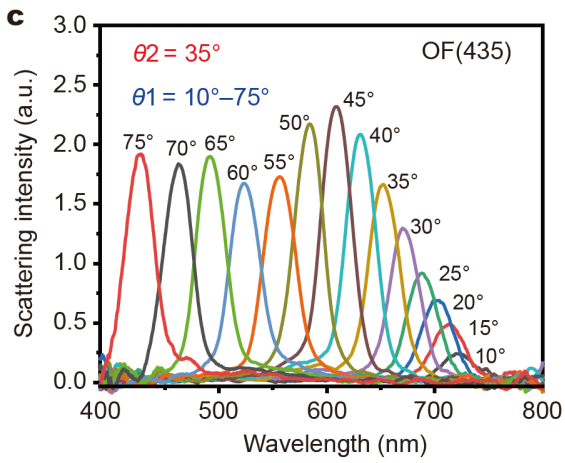

e

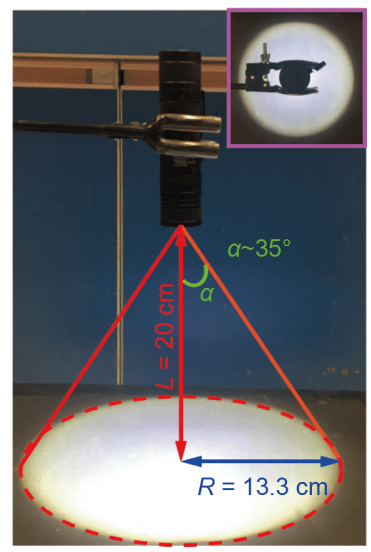

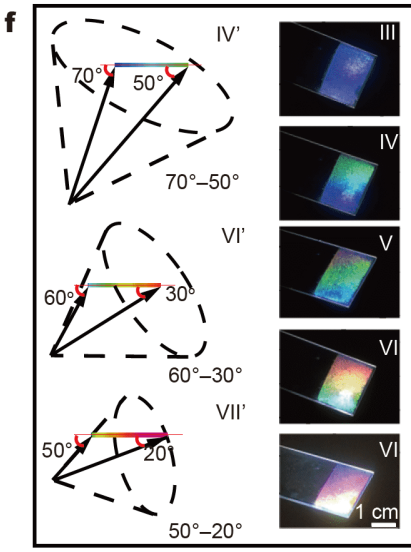

Figure 3 (a) Forward scattering spectra of $\mathrm{OF}(360)$ with $\theta 2=35^{\circ}$, and $\theta 1=10^{\circ}-65^{\circ}$. (b) Optical path diagram of the collection of forward scattering spectra. (c) Forward scattering spectra of $\mathrm{OF}$ (435) with $\theta 2=35^{\circ}$, and $\theta 1=10^{\circ}-75^{\circ}$. (d) Schematic diagram of the iridescent color scattered from OF (435) illuminated by nonparallel light. (e, f) Schematics of the experimental setup with nonparallel light (LED flashlight, beam angle $\left.=70^{\circ}\right)$ and photograph of iridescence colors (III-VII, scale bar: $1 \mathrm{~cm}$ ) with different illumination angles. (IV', VI' and VII') Photo collection angle is $35^{\circ}$.

trum rainbow colors.

The above results clearly demonstrate the incident and detecting angle dependence of scattering structural color. To quantitatively describe the scattering wavelength $(\lambda)$ versus $\theta 1$ and $\theta 2$, according to the reported result [27,34], we proposed revised equations for the OFs fabricated by $\mathrm{SiO}_{2}$ for the first time, which can be used to predict the Mie scattering spectrum position of OF:

$\lambda=N(\cos \theta 1+\sin \theta 2) D$,

$N=(1.04+0.1 \sin \theta 1)$,

where $\lambda$ is the wavelength of the scattering light and $D$ is diameter of the $\mathrm{SiO}_{2}$ spheres. $N$ is the angle dependent coefficient. The comparison between the theoretical and the experimental scattering wavelengths of OF (360) and OF (435) are listed in Tables S1, S2, respectively. The good consistence of the results proves the rationality of the proposed theoretical formula, which will provide basic guidance for choosing the right size for the wanted colors.

At the same viewing angle, iridescent structural colors can be produced by changing the incident angles, so we designed a new system to achieve scattering rainbow colors. As shown in Fig. 3d, illuminated by light with gradually changing angles, OFs produce rainbow structural colors, and the colors are determined by the initiated and terminated $\theta 1$. To obtain rainbow colors covering all spectra, OF (435) was selected here. Specifically, a point source of an LED flashlight with a beam angle of up to $70^{\circ}$ (Fig. 3e) was applied to generate consecutively changing incident angles. Interestingly, the structural colors gradually changing from blue to red (Fig. 3f III-VII) with an obvious iridescent effect were achieved by adjusting the angles and distance between the LED flashlight and the sample. Detailed angle diagrams of images IV, VI and VII are described in Fig. 3f IV', VI' and VII'. Meanwhile, the iridescent colors at different angles are consistent with the spectra listed in Fig. 3c, which shows that full spectrum colors using particles with the same diameter under the irradiation of nonparallel light can be produced. In contrast, tuning the structural colors of the most studied PCs was achieved by changing the 
size of their building blocks or lattice constant.

Based on the rainbow scattering mechanism under nonparallel light illumination, we expect that the iridescent structural colors can also be obtained in an amorphous structure, because the short-range ordered structure will also have scattering effect. Here, $435 \mathrm{~nm}$ $\mathrm{SiO}_{2}$ spheres were applied to fabricate AFs by a spraying method [14,35] to compare with the OF results. The plane SEM images and corresponding digital photos in Fig. $4 \mathrm{a}-\mathrm{c}$ show that the AF thickness can be controlled precisely by changing the volume of the $\mathrm{SiO}_{2}$ dispersion ( $w t \%=8 \%$ ) (detailed experimental parameter settings can be found in the EXPERIMENTAL SECTION). Meanwhile, the 2D-FFT pattern of AF (435)-b shown in Fig. $4 \mathrm{~d}$ with a concentric ring proves that the prepared AFs have macroscopically long-range disordered structures, which result in white body film colors under ambient light irradiation. For AF (435)-b, no obvious scattering peaks appear (Fig. S5), which shows the weak scattering ability of the amorphous structure in ambient light. Meanwhile, the scattering structural colors of an AF can be observed under LED flashlight illumination due to the irradiation intensity dependence of the scattering intensity. To verify rainbow colors of the AFs, similar operations were performed with the nonparallel light illumination, as shown in Fig. 4h. As anticipated, vivid rainbow structural colors appear (Fig. 4e-g), which are similar to that of the OF results shown in Fig. 3f. These results indicate that iridescent structural colors can also be realized in AFs. Comparing the different structures, the low coverage (Fig. 4a) of the disordered structure will result in uneven colors (Fig. 4e), while AFs with thick coverage will present a significant decrease in color brightness (Fig. 4g) due to the increased incoherent scattering. A single layer with uniform microsphere distribution will achieve the best Mie scattering intensity for the disordered structure. Meanwhile, the spraying method provides us a simple and efficient way to generate rainbow colors based on Mie scattering compared with complex designs and elaborate structures $[36,37]$.

Based on the above discussion, the full-spectrum rainbow colors originating from Mie scattering can be easily realized using $\mathrm{SiO}_{2}$ spheres with single particle size by the convenient spraying method, which greatly simplifies the structural preparation and patterning process for practical applications. In recent years, flexible and transparent displays have received great attention $[38,39]$ due to the rapid development of various smart devices. Our findings based on scattering theory contribute to this field. As displayed in Fig. 5a, an AF with the landscape pattern was first sprayed on the PET surface using a patterned template (Fig. 5b I). Vivid rainbow structural colors of the $\mathrm{PET} / \mathrm{SiO}_{2}$ were also achieved with irradiation of the point light, which agrees well with the phenomenon mentioned above. To achieve a transparent display, another layer of PDMS was infiltrated and covered on the $\mathrm{PET} / \mathrm{SiO}_{2}$ surface. After the heat curing process, a transparent composite structure $\left(\mathrm{PET} / \mathrm{SiO}_{2} / \mathrm{PDMS}\right)$ was formed (III in Fig. $5 b)$ due to the close refractive indexes of $\mathrm{SiO}_{2}$ $(n=1.46)$ and PDMS $(n=1.41)$. The transmission spectra of the sample at different stages of the sandwich structure in Fig. S6 indicate the high transparency of the final structure. The key feature of the sandwich film is that the iridescent structural color can be switched by tuning the illumination intensity. The hidden pattern (transparent) under natural light will clearly appear with light irradia-
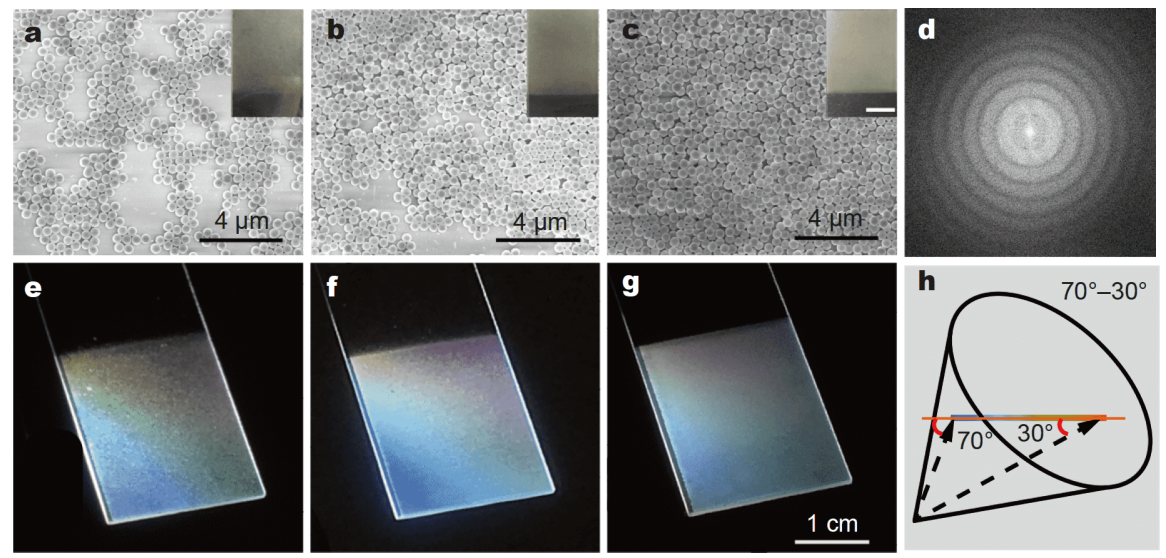

Figure 4 (a-c) Plane SEM images of the AFs assembled by $435 \mathrm{~nm} \mathrm{SiO}{ }_{2}$ particles with different thicknesses. (d) 2D-FTT pattern of sample b. (e-g) Photographs of iridescence colors of $(\mathrm{a}-\mathrm{c})$ with different thicknesses and $(\mathrm{h})$ schematic of the experimental illumination angles. All the photographs were taken at collection angle of $35^{\circ}$ (scale bar: $1 \mathrm{~cm}$ ). 


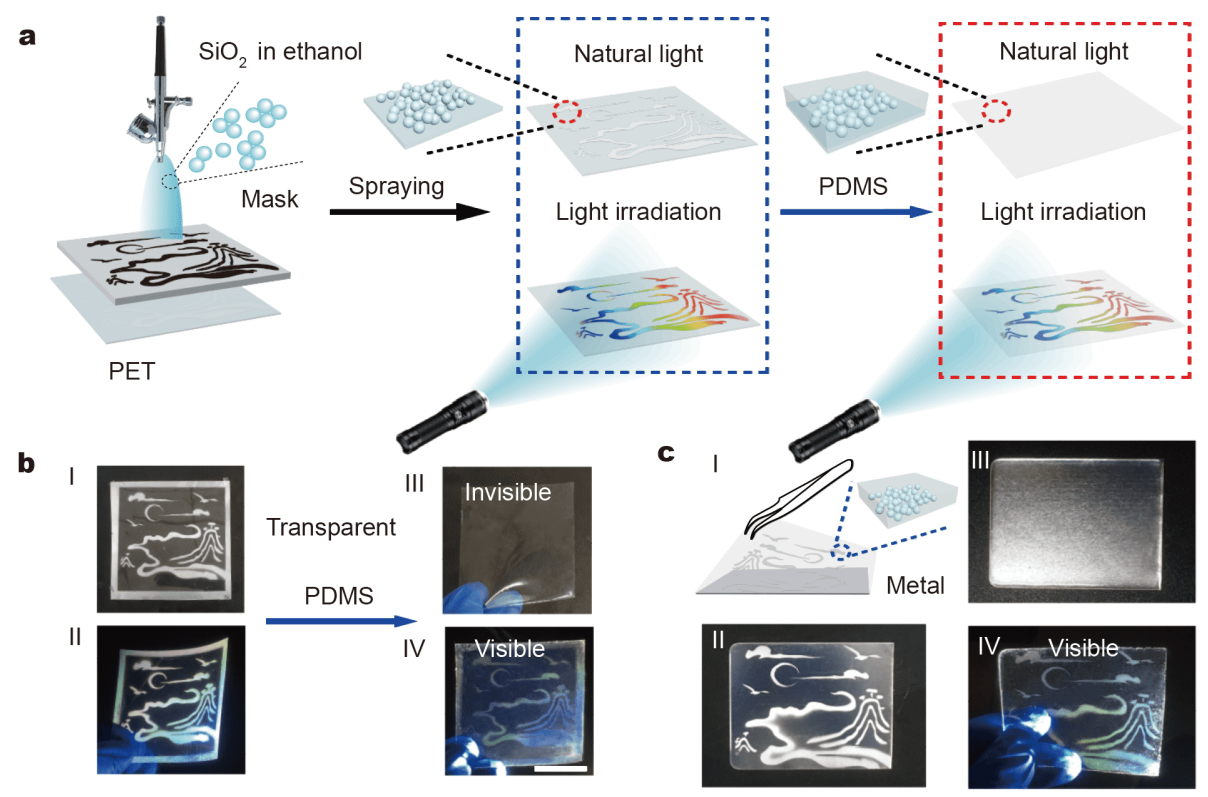

Figure 5 (a) Schematic diagram of the fabrication and generation mechanism of different structural colors with exquisite landscape patterns using the spraying method with a patterned mask on the PET substrate (scale bar: $1 \mathrm{~cm}$ ). (b) Digital images of different structural colors of PET/SiO $\mathrm{S}_{2}$ with (II) and without (I) nonparallel light illumination, and digital images of the invisible and visible patterns of $\mathrm{PET}_{\mathrm{SiO}} / \mathrm{PDMS}_{\mathrm{P}}$ freestanding films without (III) and with (IV) nonparallel light illumination (scale bar: $5 \mathrm{~cm}$ ). (c) I: Schematic diagram of preparation of the freestanding $\mathrm{PDMS}_{\mathrm{SiO}}$ film; II: digital photograph of the patterned metal substrate using the spray coating method; III: digital image of transparent structure after packaging PDMS of II; IV: transparent freestanding $\mathrm{PDMS} / \mathrm{SiO}_{2}$ film shows iridescent landscape patterns with nonparallel light illumination (scale bar: $2 \mathrm{~cm}$ ).

tion (IV in Fig. 5b).

Considering the above discussion, we know that a transparent substrate is essential for the sandwich structure, which limits our choice of matrixes for further application. Meanwhile, a sandwich structure was fabricated with different materials, and the existence of a phase interface may affect the structural stability to some extent. To obtain a uniform freestanding film, due to its excellent performance in film formation and transparency, PDMS was selected as the matrix for the transparent display, which can be peeled off easily from the hard substrate with embedded nanoparticles (Fig. 5c). Here, a steel plate was chosen as the substrate to reduce the molecular interaction between the two kinds of materials. Then, the same pattern process was performed (II in Fig. 5c). After encapsulation by PDMS, a transparent film was formed (III in Fig. 5c), which could be easily peeled off from the steel plate. It is worth noting that patterns with bright colors in the freestanding flexible transparent film can be clearly and easily observed with light illumination (Fig. 5c IV), which greatly solves the limitations for use on different substrates. Accurate pattern and color expression experimentally demonstrate the promising possibility of flexible, transparent and colorful displays based on Mie scattering.
Finally, to further prove the generality of acquiring structural color based on Mie scattering by large particles with low refractive index, PMMA spheres $(n=1.49)$ with different particle sizes were also prepared (Fig. S7). A flower-shaped AF assembled by $480 \mathrm{~nm}$ PMMA spheres on the glass substrate was created with the spraying method, as illustrated in Fig. S8. Excitingly, red, green and blue colors and gradient iridescent structural colors of the flower could be achieved easily with light illumination, which broadens the application range of microspheres and provides solid evidence for the production of structural colors based on Mie scattering in low refractive index materials.

\section{CONCLUSIONS}

In summary, we experimentally demonstrated the generation of scattering structural colors based on the Mie theory of spherical particles within a certain diameter range. Sharp scattering spectra with narrow FWHM $(24 \mathrm{~nm})$ were detected, and brilliant structural colors covering the entire visible region were generated by building ordered structures using $\mathrm{SiO}_{2}$ spheres with a diameter of $300-500 \mathrm{~nm}$. As a result, various brilliant structural colors ranging from blue to red were easily viewed under ambient light; it is worth noting that these 
same colors were not necessarily observed at the specular angle. A formula was proposed for the first time to calculate the wavelength of the Mie scattering spectrum of films assembled by $\mathrm{SiO}_{2}$ spheres. Interestingly, rainbow scattering colors based on Mie scattering were achieved by simply using an LED flashlight to illuminate the sample by adjusting the distance and angle between the LED and the sample. Moreover, because of the existence of the short-range ordered structure, rainbow colors were also easily achieved with the irradiation of point light by amorphous structures fabricated by a spraying method. More importantly, a flexible transparent rainbow-colored pattern was obtained with PET as the substrate and PDMS as the packaging material, which provides another possibility for flexible transparent displays based on Mie scattering and shows great potential in the field of display.

Received 17 April 2020; accepted 19 May 2020;

published online 27 July 2020

1 Shevtsova E, Hansson C, Janzen DH, et al. Stable structural color patterns displayed on transparent insect wings. Proc Natl Acad Sci USA, 2011, 108: 668-673

2 Vukusic P, Sambles JR. Photonic structures in biology. Nature, 2003, 424: 852-855

3 Parker AR. 515 million years of structural colour. J Opt A-Pure Appl Opt, 2000, 2: R15-R28

4 Kleyn AW, Horn TCM. Rainbow scattering from solid surfaces. Phys Rep, 1991, 199: 191-230

$5 \mathrm{Wu} \mathrm{S}$, Xia $\mathrm{H}, \mathrm{Xu}$ J, et al. Manipulating luminescence of light emitters by photonic crystals. Adv Mater, 2018, 30: 1803362

6 Takeoka Y, Yoshioka S, Takano A, et al. Production of colored pigments with amorphous arrays of black and white colloidal particles. Angew Chem Int Ed, 2013, 52: 7261-7265

7 Lee HS, Shim TS, Hwang H, et al. Colloidal photonic crystals toward structural color palettes for security materials. Chem Mater, 2013, 25: 2684-2690

8 Braun PV. Colour without colourants. Nature, 2011, 472: 423-424

9 Goerlitzer ESA, Klupp Taylor RN, Vogel N. Bioinspired photonic pigments from colloidal self-assembly. Adv Mater, 2018, 30: 1706654

10 Shang L, Zhang W, Xu K, et al. Bio-inspired intelligent structural color materials. Mater Horiz, 2019, 6: 945-958

11 Meng Y, Qiu J, Wu S, et al. Biomimetic structural color films with a bilayer inverse heterostructure for anticounterfeiting applications. ACS Appl Mater Interfaces, 2018, 10: 38459-38465

12 Marston PL, Trinh EH. Hyperbolic umbilic diffraction catastrophe and rainbow scattering from spheroidal drops. Nature, 1984, 312: 529-531

13 Zhang J, Zhu Z, Yu Z, et al. Large-scale colloidal films with robust structural colors. Mater Horiz, 2019, 6: 90-96

14 Wu S, Liu T, Tang B, et al. Structural color circulation in a bilayer photonic crystal by increasing the incident angle. ACS Appl Mater Interfaces, 2019, 11: 10171-10177

15 Zhao Z, Wang $\mathrm{H}$, Shang $\mathrm{L}$, et al. Bioinspired heterogeneous structural color stripes from capillaries. Adv Mater, 2017, 29: 1704569
16 Wu X, Hong R, Meng J, et al. Hydrophobic poly(tert-butyl acrylate) photonic crystals towards robust energy-saving performance. Angew Chem Int Ed, 2019, 131: 13690-13698

17 Li Q, Zhang Y, Shi L, et al. Additive mixing and conformal coating of noniridescent structural colors with robust mechanical properties fabricated by atomization deposition. ACS Nano, 2018, 12: 3095-3102

18 Iwata M, Teshima M, Seki T, et al. Bio-inspired bright structurally colored colloidal amorphous array enhanced by controlling thickness and black background. Adv Mater, 2017, 29: 1605050

19 Alam AM, Baek K, Son J, et al. Generating color from polydisperse, near micron-sized $\mathrm{TiO}_{2}$ particles. ACS Appl Mater Interfaces, 2017, 9: 23941-23948

20 Bai L, Mai VC, Lim Y, et al. Large-scale noniridescent structural color printing enabled by infiltration-driven nonequilibrium colloidal assembly. Adv Mater, 2018, 30: 1705667

21 Forster JD, Noh H, Liew SF, et al. Biomimetic isotropic nanostructures for structural coloration. Adv Mater, 2010, 22: 2939-2944

22 Mie G. Beiträge zur optik trüber medien, speziell kolloidaler metallösungen. Ann Phys, 1908, 330: 377-445

23 Evlyukhin AB, Novikov SM, Zywietz U, et al. Demonstration of magnetic dipole resonances of dielectric nanospheres in the visible region. Nano Lett, 2012, 12: 3749-3755

24 Retsch M, Schmelzeisen M, Butt HJ, et al. Visible Mie scattering in nonabsorbing hollow sphere powders. Nano Lett, 2011, 11: 13891394

25 García-Etxarri A, Gómez-Medina R, Froufe-Pérez LS, et al. Strong magnetic response of submicron silicon particles in the infrared. Opt Express, 2011, 19: 4815-4826

26 Baek K, Kim Y, Mohd-Noor S, et al. Mie resonant structural colors. ACS Appl Mater Interfaces, 2020, 12: 5300-5318

27 Kuznetsov AI, Miroshnichenko AE, Brongersma ML, et al. Optically resonant dielectric nanostructures. Science, 2016, 354: aag2472

28 Liu P, Yan J, Ma C, et al. Midrefractive dielectric modulator for broadband unidirectional scattering and effective radiative tailoring in the visible region. ACS Appl Mater Interfaces, 2016, 8: 22468-22476

29 Fu YH, Kuznetsov AI, Miroshnichenko AE, et al. Directional visible light scattering by silicon nanoparticles. Nat Commun, 2013, 4: 1527

30 Geffrin JM, García-Cámara B, Gómez-Medina R, et al. Magnetic and electric coherence in forward- and back-scattered electromagnetic waves by a single dielectric subwavelength sphere. Nat Commun, 2012, 3: 1171

31 Fuertes M, López-Alcaraz F, Marchi M, et al. Photonic crystals from ordered mesoporous thin-film functional building blocks. Adv Funct Mater, 2007, 17: 1247-1254

32 Bertone JF, Jiang P, Hwang KS, et al. Thickness dependence of the optical properties of ordered silica-air and air-polymer photonic crystals. Phys Rev Lett, 1999, 83: 300-303

33 Yuan W, Li Q, Zhou N, et al. Structural color fibers directly drawn from colloidal suspensions with controllable optical properties. ACS Appl Mater Interfaces, 2019, 11: 19388-19396

34 Hsiung BK, Siddique RH, Stavenga DG, et al. Rainbow peacock spiders inspire miniature super-iridescent optics. Nat Commun, 2017, 8: 2278

$35 \mathrm{Bi} \mathrm{J}, \mathrm{Wu} \mathrm{S}$, Xia H, et al. Synthesis of monodisperse single-crystal $\mathrm{Cu}_{2} \mathrm{O}$ spheres and their application in generating structural colors. J Mater Chem C, 2019, 7: 4551-4558 
Xiong K, Emilsson G, Maziz A, et al. Plasmonic metasurfaces with conjugated polymers for flexible electronic paper in color. Adv Mater, 2016, 28: 9956-9960

37 England GT, Russell C, Shirman E, et al. The optical Janus effect: Asymmetric structural color reflection materials. Adv Mater, 2017, 29: 1606876

38 Hsu CW, Zhen B, Qiu W, et al. Transparent displays enabled by resonant nanoparticle scattering. Nat Commun, 2014, 5: 3152

39 Shin S, Hwang B, Zhao ZJ, et al. Transparent displays utilizing nanopatterned quantum dot films. Sci Rep, 2018, 8: 2463

Acknowledgements This work was supported by the National Natural Science Foundation of China (21878042, 21476040 and 21276040) and the Fund for Innovative Research Groups of the National Natural Science Foundation of China Committee of Science (21421005).

Author contributions Meng Z, Wu Y, Zhang S and Wu S designed the samples; Meng $\mathrm{Z}$ performed the experiments; Meng $\mathrm{Z}$ wrote the paper with support from Wu S. All authors contributed to the general discussion.

Conflict of interest The authors declare that they have no conflict of interest.

Supplementary information Experimental details and supporting data are available in the online version of the paper.

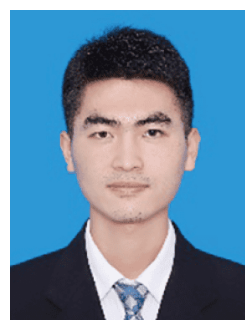

Zhipeng Meng received his bachelor degree in engineering from Qingdao University of Science and Technology in 2016, He is currently pursuing his $\mathrm{PhD}$ degree at the State Key Laboratory of Fine Chemicals at Dalian University of Technology under the supervision of Prof. Suli Wu. His research focuses on photonic crystals and structural color materials.

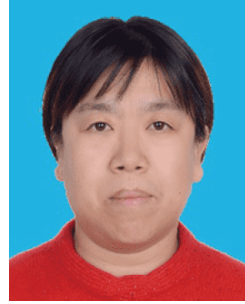

Suli Wu earned her BSc, MSc, and PhD degrees from Dalian University of Technology in 1996, 1999, and 2007, respectively. She joined the faculty of Dalian University of Technology in 2007. During 2014-2015, she worked as a visiting scholar in the group of Prof. Xiaogang Liu at the National University of Singapore. She then became a professor at Dalian University of Technology in 2017. Her interests include lanthanidedoped optical nanomaterials, quantum dots, sors, solar cells, and display devices. photonic crystals, and their applications in sen-

\section{强散射作用产生的艳丽方向性结构色}

\section{孟志鹏, 吴越, 张淑芬, 武素丽“}

摘要 通过光与微纳结构相互作用产生颜色的结构生色材料一直 是显示、防伪和刺激响应材料领域研究的热点. 与光子晶体生色 的镜面反射特性相比, 基于散射的结构生色具有更宽的可视角, 因 此受到越来越多的关注. 无序结构产生的宽散射光谱往往导致其 结构色纯度和亮度降低, 而有序结构在可见光区一般具有强的反 射和衍射作用, 因此很少有研究者关注有序结构的散射结构色. 本 研究利用粒径为 $300-500 \mathrm{~nm}$ 的 $\mathrm{SiO}_{2}$ 微球(折射率 $n=1.46$ )构建有 序结构薄膜 $(\mathrm{OF})$, 首次获得了具有窄半峰宽 (FWHM, $24 \mathrm{~nm})$ 的尖 锐散射光谱, 并提出了有序 $\mathrm{SiO}_{2}$ 薄膜散射光谱的计算公式. 有序结 构在自然光下即可以观察到鲜艳的结构色, 既无需光源照射, 也无 需在镜面角下观察. 此外, 在非平行光(点光源)照射下有序无序结 构均可产生渐变彩虹结构色. 最后, 利用单一粒径微球通过喷涂法 构筑了全光谱的结构色图案, 并利用 $\mathrm{SiO}_{2}$ 微球制备了透明复合结 构, 探索了其在柔性透明显示领域应用的可能性. 